\title{
AN EXAMPLE ON THE MAXIMAL FUNCTION ASSOCIATED TO A NONDOUBLING MEASURE
}

\author{
J. M. AldaZ
}

Abstract

We show that there is a measure $\mu$, defined on the hyperbolic plane and with polynomial growth, such that the centered maximal operator associated to $\mu$ does not satisfy weak type $(1,1)$ bounds.

\section{Introduction and main result}

Let $X$ be a metric space and let $\mu$ be a Borel measure defined on $X$. In a paper $[\mathbf{N T V}]$ that has exerted considerable influence in later developments, F. Nazarov, S. Treil, and A. Volberg showed that a good deal of the theory of Calderón Zygmund operators still holds if one replaces the doubling condition on the measure $\mu$ by the following polynomial growth condition: There exist constants $c, d$ such that for every $x \in X$ and every radius $r>0, \mu(B(x, r)) \leq c r^{d}$. Polynomial growth is a natural assumption in this area: In the euclidean case $X=\mathbb{R}^{d}$, G. David showed it is needed for the $L^{2}$ boundedness of singular integral operators that, like the Hilbert transform, are associated to kernels $K$ satisfying $|K(x, y)| \geq C|x-y|^{-d}$ (cf. [Pa, Comment 1, p. 60]).

The "Calderón Zygmund philosophy" consists in controlling singular integral operators via the maximal function, which for doubling measures is of weak type $(1,1)$. In this context it is natural to ask whether the assumption $\mu(B(x, r)) \leq c r^{d}$ can replace the doubling hypothesis and still yield weak type $(1,1)$ bounds. F. Nazarov, S. Treil, and A. Volberg bypassed this issue in [NTV], resorting instead to a modified maximal function $\tilde{M}$, where one does not take the usual average but divides by the measure of the ball with the same center and triple radius: $\tilde{M} f(x):=\sup _{r>0}(\mu(B(x, 3 r)))^{-1} \int_{B(x, r)}|f| d \mu$. This modified maximal

2000 Mathematics Subject Classification. 42B25.

Key words. Maximal function, hyperbolic plane, nondoubling measure with polynomial growth.

Partially supported by Grant BFM2003-06335-C03-03 of the D.G.I. of Spain. 
function is of weak type $(1,1)$, but in principle does not control anything. Polynomial growth is then used to show that there exist "large doubling balls" centered at every point, making it possible to utilize $\tilde{M}$ in order to find bounds for other operators.

A positive answer to the question whether the hypothesis $\mu(B(x, r)) \leq$ $c r^{d}$ is sufficient to ensure that the weak type $(1,1)$ of the centered maximal function would have allowed a development of the theory more in parallel with the classical case. The negative answer we obtain shows that such reduction is not possible. In the example we present the metric space $X$ is just the hyperbolic plane $\mathbb{H}$, with a suitably defined Borel measure. More precisely, we prove the following

Theorem. There exists a Borel measure $\mu$ on the hyperbolic plane $\mathbb{H}$ and a constant $c>0$ such that for every $w \in \mathbb{H}$ and every radius $s>0$, $\mu(B(w, s)) \leq c s$, and the centered maximal function associated to $\mu$ is not of weak type $(1,1)$.

Let us make a comment on terminology: In $[\mathbf{N T V}]$ and some later papers, a measure satisfying $\mu(B(x, r)) \leq c r^{d}$ is called $d$-dimensional. But the preceding condition does not really give a genuine notion of dimension: some measures can have uncountably many "dimensions" (for instance, planar Lebesgue measure on the unit disc), while other perfectly good measures have none (for example, the doubling measure $\mu(A):=\int_{A} x^{2} d x$ on $\mathbb{R}$, which under any reasonable definition ought to be regarded as one-dimensional). So we think it is more precise to speak of polynomial growth, as is done, for instance, in $[\mathbf{P a}]$.

This research was carried out while visiting the Universidad Autónoma de Madrid. I am indebted to the Department of Mathematics, and specially to Prof. José García-Cuerva, for the invitation, and also for several useful conversations.

\section{Proof of the theorem}

We shall utilize the upper half plane model of the hyperbolic plane $\mathbb{H}$. The following properties of this model, which can be found in geometry textbooks (see for instance, [McC, p. 237]), will be used in the proof. The hyperbolic balls $B=B_{h}(p, s)$ are also euclidean balls $B=B_{e}(w, r)$, but centers and radii vary: Namely, if $w=(a, b)$ is the euclidean center of $B$ and $r<b$ its euclidean radius, then the hyperbolic center of $B$ is $p=\left(a, \sqrt{b^{2}-r^{2}}\right)$, and the hyperbolic radius is $s=\log \sqrt{\frac{b+r}{b-r}}$.

So as a topological space, our set $X$ is just $\left\{(x, y) \in \mathbb{R}^{2}: y>0\right\}$ with the usual topology, metrized by the hyperbolic distance. We define 
a Borel measure $\mu$ on $X=\mathbb{H}$ as follows: let $m_{1}$ be the restriction to the upper half plane of the usual Gaussian probability on $\mathbb{R}^{2}$, let $d m_{2}:=\chi_{A} d w$, where $d w$ stands for planar Lebesgue measure and $A:=$ $\left\{(x, y) \in \mathbb{R}^{2}: x>1, x^{-1}>y>0\right\}$, and finally, set $\mu:=m_{1}+m_{2}$.

First we show that

$$
M_{\mu} f(w):=\sup _{s>0} \frac{1}{\mu B_{h}(w, s)} \int_{B_{h}(w, s)}|f| d \mu
$$

is not of weak type $(1,1)$. By the usual approximation argument via convolutions, we may use a Dirac delta instead of a function. So consider $\delta_{(R+1 / 2,1)}$, where $R \gg 0$. We will see that

$$
\mu\left\{M_{\mu} \delta_{(R+1 / 2,1)}(w)>\frac{(R-1)^{3 / 2}}{3}\right\}>\frac{1}{2 R},
$$

from which the result follows by letting $R \rightarrow \infty$. Since

$$
\begin{aligned}
\mu\left\{(x, y) \in \mathbb{R}^{2}\right. & \left.: R<x<R+1,0<y<x^{-1}\right\} \\
& >m_{2}\left\{(x, y) \in \mathbb{R}^{2}: R<x<R+1,0<y<x^{-1}\right\} \\
& =\log \left(1+\frac{1}{R}\right)>\frac{1}{2 R},
\end{aligned}
$$

it is enough to prove that

$$
\begin{aligned}
\left\{(x, y) \in \mathbb{R}^{2}: R<x<R+1,\right. & \left.0<y<x^{-1}\right\} \\
& \subset\left\{M_{\mu} \delta_{(R+1 / 2,1)}(w)>\frac{(R-1)^{3 / 2}}{3}\right\} .
\end{aligned}
$$

Fix $(x, y)$ such that $R<x<R+1,0<y<x^{-1}$, and select $r \in[1 / 2,1)$ so that the hyperbolic center of $B_{e}((x, 1), r)$ is $(x, y)$. Since $B_{e}((x, 1), r)$ contains the point $(R+1 / 2,1)$,

$$
M_{\mu} \delta_{(R+1 / 2,1)}((x, y)) \geq \frac{1}{\mu B_{e}((x, 1), r)} \geq \frac{1}{\mu B_{e}((R, 1), 1)} .
$$

To estimate $\mu B_{e}((R, 1), 1)$, note first that

$$
m_{1} B_{e}((R, 1), 1)<\int_{R-1}^{\infty} e^{-t^{2} / 2} d t<\int_{R-1}^{\infty} \frac{t}{R-1} e^{-t^{2} / 2} d t=\frac{e^{-(R-1)^{2} / 2}}{R-1} .
$$

Suppose next that $(x-R)^{2}+(y-1)^{2}<1$ and $0<y<x^{-1}$. Solving for $y$ in $(x-R)^{2}+(y-1)^{2}<1$ we get

$$
\frac{(x-R)^{2}}{2}<1-\sqrt{1-(x-R)^{2}}<y<x^{-1}<\frac{1}{R-1} .
$$


So

$$
\begin{aligned}
& \left\{(x, y) \in \mathbb{R}^{2}:(x-R)^{2}+(y-1)^{2}<1,0<y<x^{-1}\right\} \\
& \subset\left\{(x, y) \in \mathbb{R}^{2}: R-\sqrt{\frac{2}{R-1}}<x<R+\sqrt{\frac{2}{R-1}}, 0<y<(R-1)^{-1}\right\},
\end{aligned}
$$

whence

$$
m_{2} B_{e}((R, 1), 1)<\int_{0}^{\frac{1}{R-1}} \int_{R-\sqrt{\frac{2}{R-1}}}^{R+\sqrt{\frac{2}{R-1}}} d x d y=\frac{2 \sqrt{2}}{(R-1)^{3 / 2}} .
$$

Thus, by taking $R$ sufficiently large,

$$
\mu B_{e}((R, 1), 1)<\frac{3}{(R-1)^{3 / 2}},
$$

and it follows that $M_{\mu}$ is not of weak type $(1,1)$.

Next we prove that there is a constant $c>0$ such that for all $w \in \mathbb{H}$, $\mu B_{h}(w, s) \leq c s$. We find constants $c_{1}, c_{2}>0$ with $m_{1} B_{h}(w, s) \leq c_{1} s$ and $m_{2} B_{h}(w, s) \leq c_{2} s$ for all $w \in \mathbb{H}$. From now on we shall adhere to the convention whereby a constant $c$ may change its value from one line to the next.

Recall that we use $B_{e}((a, b), r)=B_{h}\left(\left(a, b^{\prime}\right), s\right)$ to denote the same ball $B \subset \mathbb{H}$, with respect to the euclidean metric in the first case and the hyperbolic metric in the second. Here

$$
s=\frac{1}{2} \log \left(1+\frac{2 r}{b-r}\right) .
$$

For small values of $b$, and therefore of $r, s$ controls $r$, so $m_{1}$ and $m_{2}$ can simply be replaced by planar Lebesgue measure to prove the polynomial growth. More precisely, suppose $0<b \leq 3$. Then $0<r<3$ and there exists a $c>0$ such that $s \geq c r$. So for $i=1,2$,

$$
m_{i} B_{h}\left(\left(a, b^{\prime}\right), s\right)=m_{i} B_{e}((a, b), r) \leq c r \leq c^{\prime} s .
$$

Suppose next that $b>3$. Then $m_{2} B_{e}((a, b), r)=0$ unless $r>b-1$. In this case we have

$m_{2} B_{h}\left(\left(a, b^{\prime}\right), s\right)=m_{2} B_{e}((a, b), r) \leq \log (1+2 r) \leq \log \left(1+\frac{2 r}{b-r}\right)=2 s$.

Finally, to prove the polynomial growth of $m_{1}$ on $\{b>3\}$ we consider the following cases. If $r$ and $b$ are comparable, say, $b / 3 \leq r<b$, everything is trivial, since

$$
m_{1} B_{h}\left(\left(a, b^{\prime}\right), s\right)=m_{1} B_{e}((a, b), r) \leq 1 / 2
$$

and

$$
s \geq \frac{1}{2} \log 2 .
$$


So suppose $0<r<b / 3$. Then

$$
\begin{aligned}
m_{1} B_{h}\left(\left(a, b^{\prime}\right), s\right) & =m_{1} B_{e}((a, b), r) \leq m_{1} B_{e}((0, b), r) \\
& \leq \frac{1}{2 \pi} \int_{-r}^{r} \int_{b-r}^{\infty} e^{-\frac{x^{2}+y^{2}}{2}} d y d x \\
& \leq \frac{1}{2 \pi} \frac{e^{-\frac{(b-r)^{2}}{2}}}{b-r} \int_{-r}^{r} e^{-\frac{x^{2}}{2}} d x \\
& \leq \frac{e^{-\frac{(b-r)^{2}}{2}}}{b-r} \min \{1,2 r\} .
\end{aligned}
$$

Now if $0<r<1 / 2$, then

$$
\frac{e^{-\frac{(b-r)^{2}}{2}}}{b-r} 2 r \leq \frac{c 2 r}{b} \leq c^{\prime} \frac{1}{2} \log \left(1+\frac{2 r}{b}\right) \leq c^{\prime} s,
$$

while if $1 / 2 \leq r<b / 3$, then

$$
\frac{e^{-\frac{(b-r)^{2}}{2}}}{b-r} \leq \frac{c^{\prime}}{b} \leq c^{\prime \prime} \frac{1}{2} \log \left(1+\frac{1}{b}\right) \leq c^{\prime \prime} s .
$$

Remark. One might ask for which locally finite Borel measures $\mu$ on $\mathbb{H}$ is the centered maximal function $M_{\mu}$ of weak type $(1,1)$. Locally finite means that for every $x \in \mathbb{H}$ there is an open neighborhood of $x$ with finite measure. This implies that compact sets and balls have finite measure. If $\mu$ has compact support (and hence it is finite), then it follows from Besicovitch's covering theorem that $M_{\mu}$ is of weak type $(1,1)$. But being finite, even in the presence of polynomial growth, is not enough to ensure the weak type of $M_{\mu}$. The example we present above can be easily modified so that $\mu(\mathbb{H})<\infty$ : Instead of using the set $\left\{(x, y) \in \mathbb{R}^{2}\right.$ : $\left.x>1, x^{-1}>y>0\right\}$ to define $m_{2}$, take for instance $A:=\left\{(x, y) \in \mathbb{R}^{2}\right.$ : $\left.x>0, e^{-x}>y>0\right\}$, and argue as before.

On the other hand, the centered maximal function associated to area in the hyperbolic plane is of weak type $(1,1)$ (cf. $[\mathbf{S t r}]$ ), even though area does not satisfy any polynomial growth condition. So there seems to be no significant relationship between the weak type of the maximal operator and the polynomial growth of the underlying measure.

To finish, we mention that while the doubling condition on the measure is sufficient to ensure the weak type of the maximal function, for $\mathbb{H}$ this is irrelevant: It follows from the remark in p. 67 of $[\mathbf{C W}]$ together with Example 3.5.2 of [Luu] that the hyperbolic plane admits no doubling measures (in particular, area is not doubling).

The referee points out that one can relax the doubling condition on a measure $\mu$, by requiring that it hold only at the points of its support, and 
this weaker version also entails the weak type of the maximal operator. With this definition there are plenty of "doubling measures" on $\mathbb{H}$, so one might object to the assertion that the doubling condition on the measure is irrelevant for $\mathbb{H}$. However, this weakened version of doubling ignores in an essential way the geometry of $\mathbb{H}$. For instance, let $\mu$ be the linear Lebesgue measure on $\{(x, 1): x \in \mathbb{R}\} \subset \mathbb{H}$. This measure is certainly doubling in the weaker sense; but I would be inclined to think that if one works with $\mu$ one is doing analysis on the real line rather than on the hyperbolic plane. In fact, any such weakly doubling measure must live on a set with geometry substantially different from that of $\mathbb{H}$.

\section{References}

[CW] R. R. CoIfman AND G. WeIss, "Analyse harmonique noncommutative sur certains espaces homogènes. Étude de certaines intègrales singulières", Lecture Notes in Mathematics 242, Springer-Verlag, Berlin-New York, 1971.

[Luu] J. LuUkKainen, Assouad dimension: antifractal metrization, porous sets, and homogeneous measures, J. Korean Math. Soc. 35(1) (1998), 23-76.

[McC] J. MCClEARY, "Geometry from a differentiable viewpoint", Cambridge University Press, Cambridge, 1994.

[NTV] F. NAZARov, S. Treil AND A. VolberG, Weak type estimates and Cotlar inequalities for Calderón-Zygmund operators on nonhomogeneous spaces, Internat. Math. Res. Notices 9 (1998), 463-487.

[Pa] H. PAJOT, "Analytic capacity, rectifiability, Menger curvature and the Cauchy integral", Lecture Notes in Mathematics 1799, Springer-Verlag, Berlin, 2002.

[Str] J.-O. StrömberG, Weak type $L^{1}$ estimates for maximal functions on noncompact symmetric spaces, Ann. of Math. (2) 114(1) (1981), 115-126.

Departamento de Matemáticas y Computación

Universidad de La Rioja

26004 Logroño, La Rioja

Spain

E-mail address: aldaz@dmc.unirioja.es

Primera versió rebuda el 25 de gener de 2005, darrera versió rebuda el 10 de març de 2005. 